This is a non-peer reviewed pre-print submitted to EarthArxiv. Subsequent peer-reviewed versions f this manuscript may have slightly different content.

The authors welcome feedback.

Please contact Sandy H. S. Herho (herho@umd.edu) regarding this manuscript's content. 


\title{
On the statistical learning analysis of rain gauge data over the Natuna Islands
}

\author{
Sandy H. S. Herho \\ Department of Geology, \\ College of Computer, Mathematical, and Natural Sciences, \\ University of Maryland, \\ College Park, MD, USA 20742 \\ herho@umd.edu \\ Faiz R. Fajary \\ Atmospheric Science Research Group, \\ Faculty of Earth Sciences and Technology, \\ Bandung Institute of Technology, \\ Bandung, West Java, Indonesia 40132 \\ faiz@fitb.itb.ac.id \\ Dasapta E. Irawan \\ Applied Geology Research Group, \\ Faculty of Earth Sciences and Technology, \\ Bandung Institute of Technology, \\ Bandung, West Java, Indonesia 40132 \\ r-win@office.itb.ac.id
}

\begin{abstract}
This article presents state-of-the-art statistical learning methods for analyzing rain gauge data over the Natuna Islands. By using shape preserving piecewise cubic interpolation, we managed to interpolate 671 null values from the daily precipitation data. Dominant periodicity analysis of daily precipitation signals using Lomb-Scargle Power Spectral Density shows annual, intraseasonal, and interannual precipitation patterns over the Natuna Islands. Unsupervised anomaly analysis using the Isolation Forest algorithm shows there are 146 anomaly daily precipitation data points. We also conducted an experiment to predict the accumulation of monthly precipitation over the Natuna Islands using the Bayesian structural time series algorithm. The results show that the local linear trend with seasonality model is able to model the value of accumulated monthly precipitation for a twelve-month prediction horizon. The work presented here has profound implications for rainfall observations in this area.

key words: observational tropical meteorology, cubic interpolation, LombScargle PSD, isolation forest, Bayesian structural time series
\end{abstract}

1 Introduction

Natuna Islands are an archipelago which is administratively located in Natuna Regency, Riau Islands Province, Indonesia. Astronomically, the Natuna Islands are located at $3^{\circ} \mathrm{N}$ to $4^{\circ} 46^{\prime} \mathrm{N}$ and $107^{\circ} 45^{\prime} \mathrm{E}$ to $108^{\circ} 23^{\prime} E$. Directly adjacent to the South China Sea to the east and north, making the Natuna Islands one of the front lines of Indonesia's territorial defense in an area rich in natural resources

Preprint. Under review. 
that has the potential to cause conflict on a regional scale [Johnson, 1997, Sudirman et al., 2013. Kurniaty et al. 2018|. One way to strengthen the defense system in an area is to know in detail the intelligence data, one of which is meteorological data [Tuite and Harley, 2013, Azhari et al., 2021]. Therefore, knowledge of rainfall data in the Natuna Islands plays a vital role in Indonesia's defense system in the South China Sea. To deal with this problem, we applied some of the recently developed statistical learning techniques [Vapnik, 1999] to the daily rain gauge data from the BMKG Ranai meteorological station (Figure 1), which is the only meteorological station in the Natuna Islands, from 01 January 2013 to 31 December 2020 in this study.

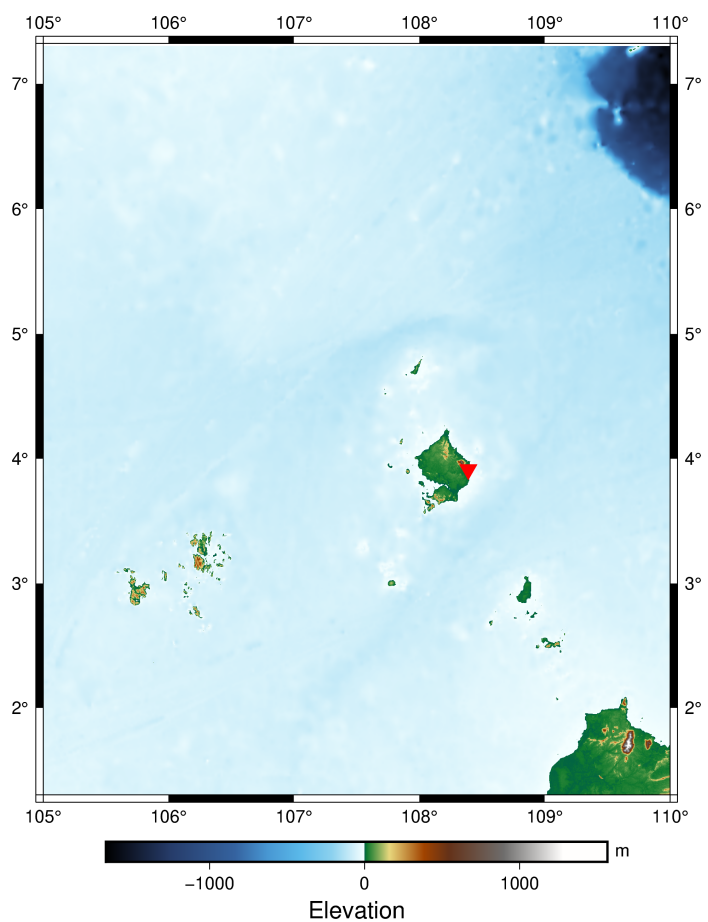

Figure 1: Topography of the Natuna Islands (rendered using PyGMT [Uieda et al. 2021]). Red triangle denotes BMKG Ranai meteorological station at $3^{\circ} 54^{\prime} 43^{\prime \prime} \mathrm{N}, 108^{\circ} 23^{\prime} 35^{\prime \prime} \mathrm{E}$.

\section{Data interpolation}

The presence of 671 null values from a total of 2892 data points can certainly complicate the processing of our daily precipitation data. Therefore, data interpolation is needed as a prerequisite for the next stage of data processing. We use shape-preserving piecewise cubic interpolation [Wolberg and Alfy, 1999| to interpolate daily precipitation over the Natuna Islands. This algorithm aims to model data points into a third degree polynomial of $P(x)$ with coefficients $[a, b, c, d]$ at intervals $\left[x_{1}, x_{2}\right]$ as follows,

$$
P(x)=a\left(x-x_{1}\right)^{3}+b\left(x-x_{1}\right)^{2}+c\left(x-x_{1}\right)+d
$$

On each subinterval $x_{k} \leq x \leq x_{k+1}$, the polynomial $P(x)$ is a cubic Hermite interpolating polynomial for the given data points with specified derivatives at the interpolation points. $P(x)$ interpolates $y$, that is, $P\left(x_{j}\right)=y_{j}$, and the first derivative $P^{\prime}(x)$ is continuous. The second derivative $P^{\prime \prime}(x)$ is probably not continuous so jumps at the $x_{j}$ are possible. The cubic interpolant $P(x)$ is shape preserving. The slopes at the $x_{j}$ are chosen in such a way that $P(x)$ preserves the shape of the data and respects monotonicity. Therefore, on intervals where the data is monotonic, so is $P(x)$, and at points where the data has a local extremum, so does $P(x)$. To simplify the computation process, we use pandas [McKinney, 2010] built-in interpolate method with the pchip option. The results of the interpolation are shown in Figure 2 as follows, 


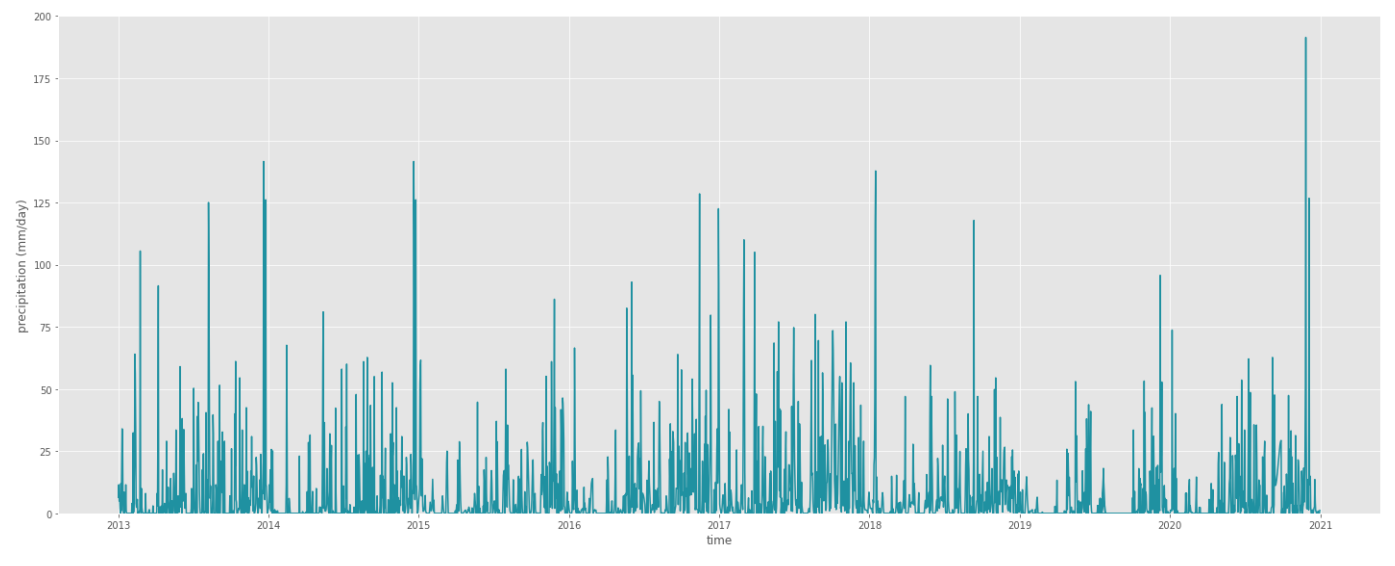

Figure 2: Result from shape-preserving piecewise cubic interpolation of daily precipitation data.

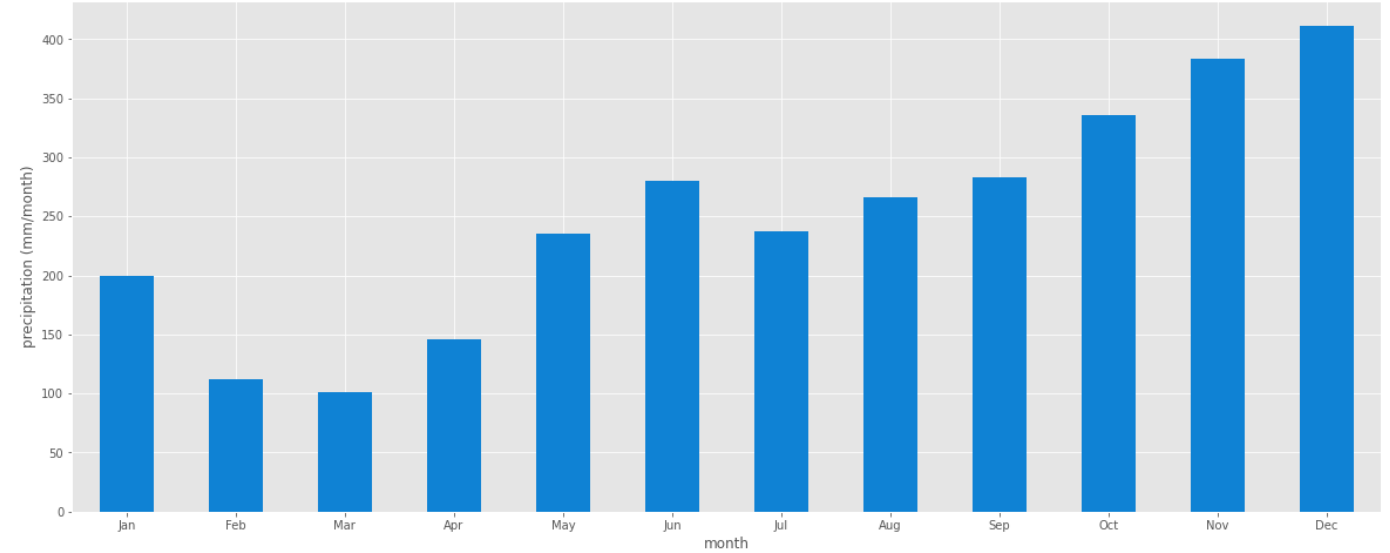

In order to determine the precipitation patterns in the Natuna Islands, we average the accumulated monthly rainfall as shown in Figure 3. The result shows that there are two peak periods of rainfall in September to December (SOND) and May to July (MJJ). Natuna Islands can be categorized as region $\mathrm{B}$ in the classification of rainfall areas over the Indonesian Maritime Continent (IMC) described by Aldrian and Susanto [2003]. These two peaks of rainfall were the result of a change in the direction of meridional movement of the Inter Tropical Convergence Zone (ITCZ) [Davidson et al., 1984, Aldrian and Susanto, 2003].

Figure 3: The annual cycle of the accumulated monthly precipitation over the Natuna Islands.

\section{Periodicity analysis}

We use the Lomb-Scargle Power Spectral Density (PSD) [Lomb, 1976, Scargle, 1983, Trauth, 2015] to quantitatively analyze the dominant periodicities of precipitation over the Natuna Islands. Assuming daily precipitation data as $y(t)$ of $N$ days, normalized Lomb-Scargle periodogram $P_{x}(\omega)$, as a function of angular frequency $\omega=2 \pi f>0$, is calculated using the following equation,

$$
P_{x}(\omega)=\frac{1}{2 s^{2}}\left\{\frac{\left[\sum_{j}\left(y_{i}-\bar{y}\right) \cos \omega\left(t_{j}-\tau\right)\right]^{2}}{\sum_{j} \cos ^{2} \omega\left(t_{j}-\tau\right)}+\frac{\left[\sum_{j}\left(y_{i}-\bar{y}\right) \sin \omega\left(t_{j}-\tau\right)\right]^{2}}{\sum_{j} \sin ^{2} \omega\left(t_{j}-\tau\right)}\right\}
$$

57 , where the arithmetic mean $\bar{y}$ and variance $s^{2}$ are respectively expressed by equations (3) and (4) as 58 follows,

$$
\bar{y}=\frac{1}{N} \sum_{i=1}^{N} y_{i}
$$




$$
s^{2}=\frac{1}{N-1} \sum_{i=1}^{N}\left(y_{i}-\bar{y}\right)^{2}
$$

59

While the constant $\tau$ is defined through the following trigonometric relationship,

$$
\tan (2 \omega \tau)=\frac{\sum_{j} \sin 2 \omega t_{j}}{\sum_{j} \cos 2 \omega t_{j}}
$$

To evaluate the power spectra produced by these calculations, we use the False-Alarm probability $P(>z)$ of the null hypothesis, i.e. the probability that given peaks in periodogram are not significant which is defined as follows,

$$
P(>z) \equiv 1-\left(1-e^{-z}\right)^{M}
$$

where $M$ is the number of independent frequencies. We use Nyquist criterion, suggested by Press et al. [2007] to determine the number of $M$. We do this calculation and evaluation using built-in functions available in MATLAB ${ }^{\circledR}$ computing environment. The results are shown in Figure 4 as follows,

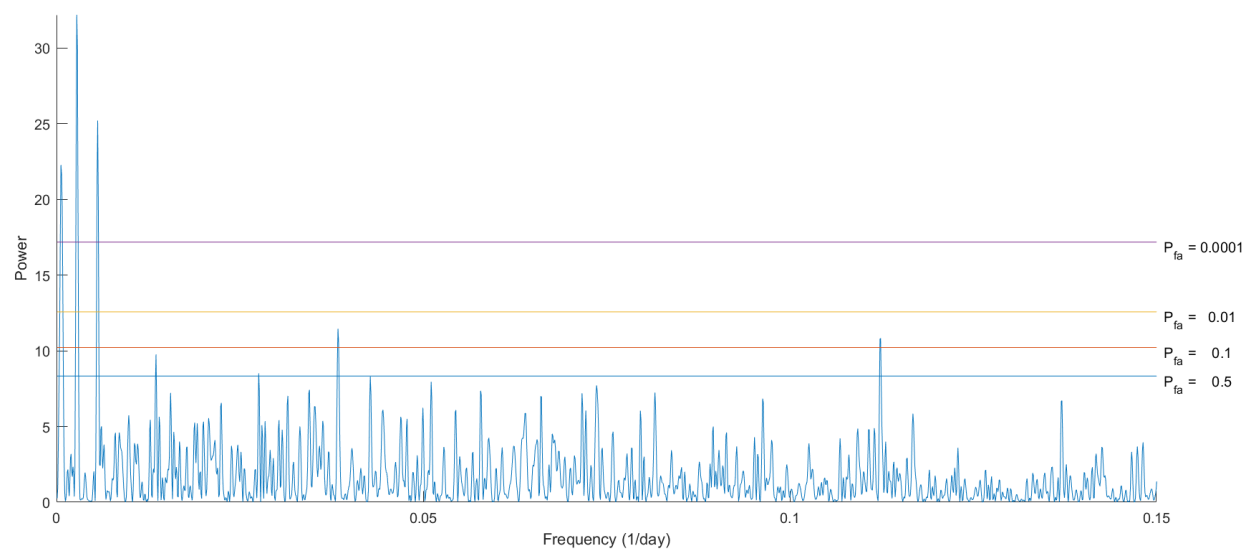

Figure 4: Lomb-Scargle PSD with the false-alarm probabilities (horizontal lines). The plot suggests that the $361.5,177.9,1652.6$ day cycles are highly significant.

There are three dominant rainfall periodicity peaks in the Natuna Islands, i.e. annual (361.5 days), intraseasonal (177.9 days), and interannual (1652.6 days) patterns. These dominant patterns are consistent with several studies that were conducted in region B [Aldrian and Susanto, 2003, Xavier et al. 2020, Narulita et al. 2021]. Based on a study conducted on Bintan Island, $538 \mathrm{~km}$ southwest of Ranai, by |Narulita et al. [2021], it is known that the annual pattern can be caused by ITCZ oscillation. Meanwhile, the intraseasonal pattern is caused by a combination of Madden-Julian Oscillation (MJO), Borneo vortex, and the cold surge phenomena. El Niño-Southern Oscillation (ENSO) and Indian Ocean Dipole (IOD) are considered to affect interannual rainfall pattern in the region.

\section{Anomaly detection}

The presence of anomalies in the Automatic Weather Station (AWS) rain gauge observations can be caused by various factors such as sensor malfunction, hardware error, power supply error, ambient environment change, and abnormal weather phenomena [Lee et al., 2018]. With advances in technology, this anomaly detection process can be done automatically by applying the anomaly detection 
methods used in the field of statistical learning. In this section, we try to experiment by applying an anomaly detection algorithm to rainfall measurements in the Natuna Islands without assuming data linearity using the Isolation Forest (iForest) algorithm.

iForest [Liu et al. 2012] is an algorithm that is widely used to perform anomaly detection on timeseries data [Calheiros et al. 2017, Puggini and McLoone, 2018, Qin and Lou, 2019, Zhong et al. 2019. Li and Jung. 2021|. The algorithm is based on the fact that there are data points that are few and very different from the dominant data points, then based on this assumption, it can be explained that anomalies are susceptible to a mechanism called isolation. This method is very useful because it fundamentally introduces the use of isolation trees as an effective way of detecting anomalies from datasets. In addition, this method can work with low linear time complexity and low memory requirements so it can perform well regardless of data size [Yao et al. 2019]. The main idea of the iForest is that the number of data points is abnormal is usually small, and there is a significant difference between normal and these abnormal attributes. This algorithm has the same basic pattern as the available decision tree model break down the complex decision-making process into more simple, so that the decision-making process would be more interpreted to find the solution to the problem [Zhang et al. 2019]. iForest is also an efficient way to detect anomalies in high-dimensional datasets. One can make observations by randomly selecting features and then selecting the split values between the maximum and minimum values of the features selected using this algorithm [Qin and Lou, 2019, Yao et al., 2019].

We use iForest as an unsupervised ensemble classifier on daily precipitation data. The score used to classify anomalies $s(x, n)$ is calculated using the equation (7) below,

$$
s(x, n)=2^{-\frac{E(h(x)}{c(n)}}
$$

,where $h(x)$ is the number of edges of isolation trees for point $x, c(n)$ is a normalization constant, and $E(h(x))$ is the average of $h(x)$ from a collection of isolation trees. Because we use iForest as an unsupervised classifier, the threshold values do not need to be specified. We use PyCaret library Ali [2020] in the Python computing environment to automatically compute the iForest algorithm.

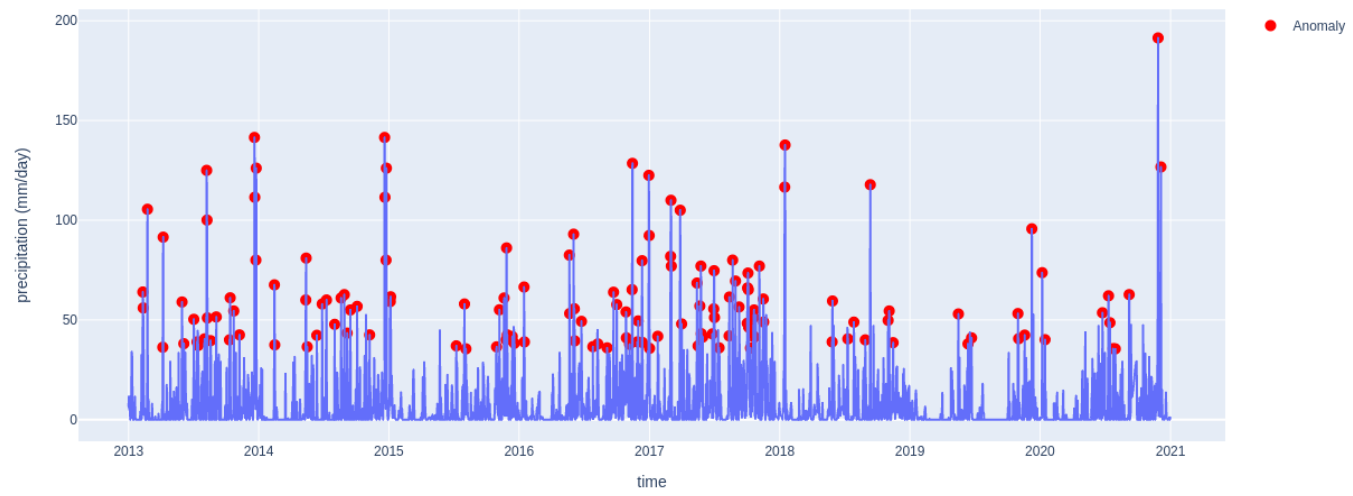

Figure 5: Unsupervised iForest anomaly detection in the interpolated daily precipitation data over the Natuna Islands.

146 anomalous data points are presented in red dots in Figure 5. It can be seen in that figure, that all anomalies occurred in relatively high rainfall values. There are three possibilities that cause data points to be detected as anomaly, namely due to measurement errors, natural anomalous events, or artifacts due to interpolation errors. Therefore, further investigation is needed on these iForest anomalies.

\section{Probabilistic forecasting}

In this section, we try to make a one-step-ahead forecasting to predict the accumulation of monthly rainfall over the Natuna Islands in the next twelve months time horizon. We use a state-space prob- 


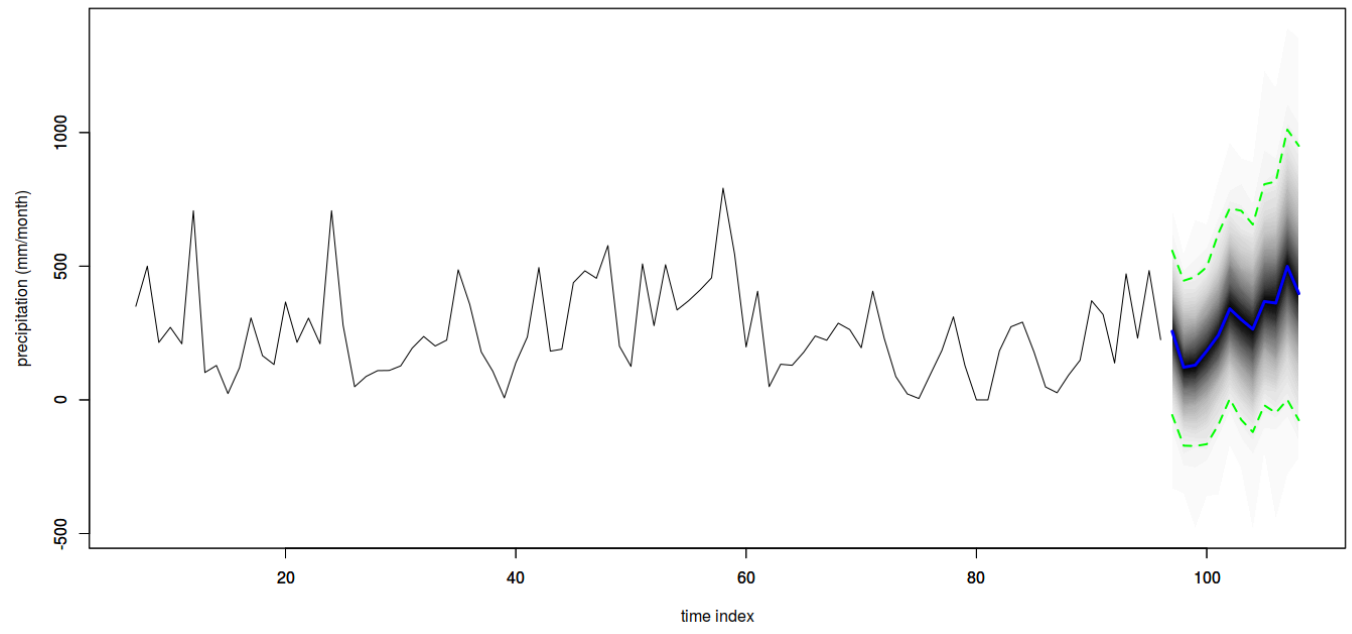

, where $\tau_{t}$ is the seasonal component, $s$ is the dummy variables which amount to one in each season, and $\phi_{t}$ is the local linear trend model which is described in equation (9) as follows,

$$
\begin{aligned}
y_{t}=\mu_{t}+\varepsilon_{t}, & \varepsilon_{t} \sim \mathcal{N}\left(0, \sigma_{\varepsilon}^{2}\right) \\
\mu_{t+1}=\mu_{t}+\nu_{t}+\xi_{t}, & \xi_{t} \sim \mathcal{N}\left(0, \sigma_{\xi}^{2}\right) \\
\nu_{t+1}=\nu_{t}+\zeta_{t}, & \zeta_{t} \sim \mathcal{N}\left(0, \sigma_{\zeta}^{2}\right)
\end{aligned}
$$

where $\mu_{t}$ is the unobserved state and $\nu_{t}$ is the additional state component which is the slope of the trend. We use the error variance distribution parameters to calculate the prior of this model. Numerical computations are used to estimate the Kalman filter, Kalman smoother, and Markov Chain Monte Carlo (MCMC) sampler on the posterior distribution. The whole computing process is done automatically by using the bsts [Scott, 2020] package in the R computing environment. The results of the probabilistic predictions for the next twelve months are shown in Figure 6 as follows, In evaluating the model, we use one-step-ahead error prediction as follows,

Figure 6: Predicted accumulation of monthly precipitation for twelve-month horizon. The solid blue line indicates the median of the prediction results, the green dotted line indicates the $95 \%$ credible interval, and the gray shading area indicates the posterior density distribution.

$$
\text { error }=y_{t}-\mathbb{E}\left(y_{t} \mid y_{1}, \cdots, y_{t-1} ; \theta\right)
$$

, where the values of $y_{1}, \cdots, y_{t-1}$ and the model parameter $\theta$ are fixed at the current values in the MCMC algorithm. Cummulative total of the mean absolute errors for each univariate BSTS models, local level; local linear trend; local linear trend with seasonal components, are shown in the Figure. The results show that local linear trend with seasonal components is the best model configuration choice among the three univariate BSTS models, with an error curve that is relatively not steep over time. 


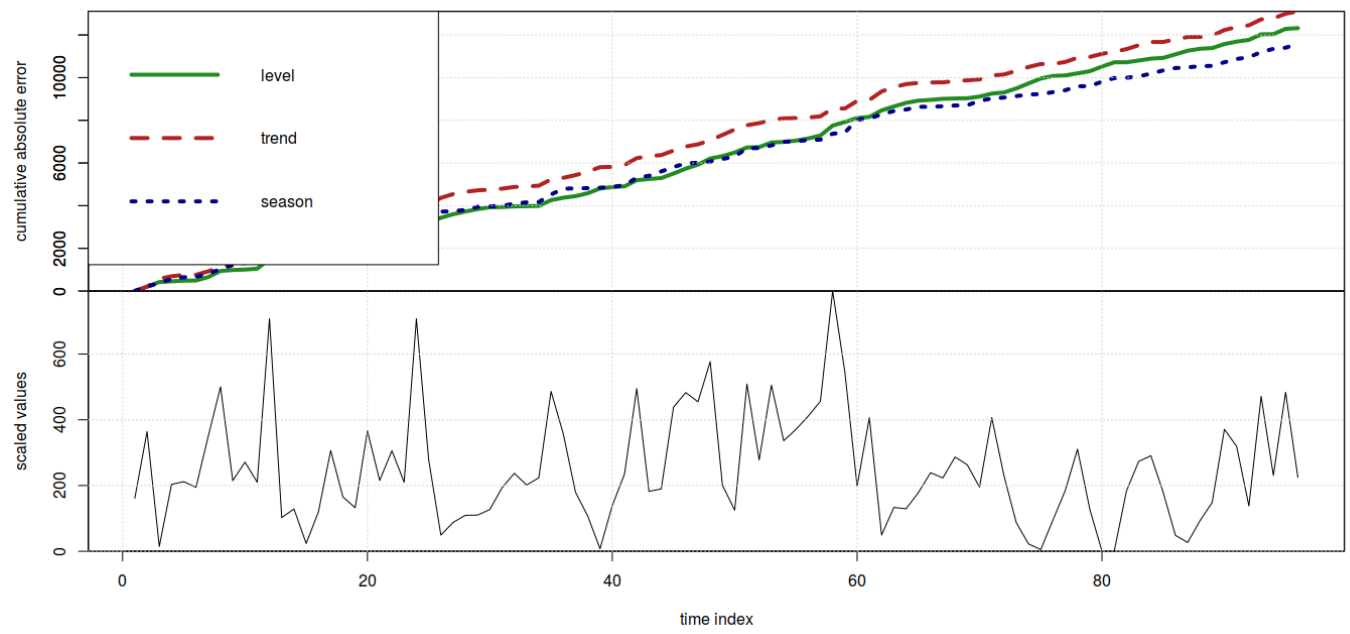

Figure 7: Heuristic comparison of univariate BSTS models in terms of cumulative prediction error for the accumulation of monthly precipitation over the Natuna Islands.

\section{Concluding remarks and future work suggestion}

We have applied some of the latest techniques in statistical learning, namely interpolation, signal processing, anomaly analysis, and probabilistic prediction, to assist the process of analyzing rain gauge station data from the Natuna Islands. Our study suggests possible extension of the work by using nonlinear interpolation techniques, nonstationary signal processing, matching anomaly analysis from iForest with other data sources, and considering related climatic phenomena as regressors in the future BSTS framework, with adequate computational resources, developed as a probabilistic prediction of daily rainfall by optimizing the computational time of the MCMC sampler.

\section{Acknowledgements}

The authors are thankful to Cristy Q. Ho for her critical and constructive comments on the early drafts of this manuscript. The authors gratefully thank Herho Group Corp., Rialto, CA and P3MI ITB for funding the publication and dissemination stage. The code and datasets used for this work are posted on GitHub at https://github.com/sandyherho/natunaRainStatAnal.

\section{Conflicts of Interest}

The authors declare no conflict of interest.

\section{Author's contribution}

SHSH: formulating ideas, write the codes and drafting the manuscript, FRF: formulating ideas and drafting the manuscript, DEI: formulating ideas and drafting the manuscript.

\section{References}

E. Aldrian and R. D. Susanto. Identification of three dominant rainfall regions within indonesia and their relationship to sea surface temperature. International Journal of Climatology, 23(12): 1435-1452, 2003. doi: https://doi.org/10.1002/joc.950.

M. Ali. PyCaret: An open source, low-code machine learning library in Python, July 2020. URL https://www. pycaret.org. PyCaret version 2.3. 
F. Azhari, N. B. Sukoco, and K. I. Fatoni. Studi karakteristik parameter meteorologi dan gelombang untuk operasi amfibi di perairan singkawang kalimantan barat (study of the characteristics of meteorological and wave parameters for amphibious operations in singkawang, west kalimantan). Jurnal Chart Datum, 6(1):1-9, 2021. doi: https://doi.org/10.37875/chartdatum.v6i1.169.

R. N. Calheiros, K. Ramamohanarao, R. Buyya, C. Leckie, and S. Versteeg. On the effectiveness of isolation-based anomaly detection in cloud data centers. Concurrency and Computation: Practice and Experience, 29(18):e4169, 2017. doi: https://doi.org/10.1002/cpe.4169.

N. E. Davidson, J. L. Mcbride, and B. J. McAvaney. Divergent circulations during the onset of the 1978-79 australian monsoon. Monthly Weather Review, 112(9):1684 - 1696, 1984. doi: 10.1175/1520-0493(1984)112〈1684:DCDTOO $\rangle 2.0 . C O ; 2$.

D. Johnson. Drawn into the fray: Indonesia's natuna islands meet china's long gaze south. Asian Affairs: An American Review, 24(3):153-161, 1997. doi: 10.1080/00927679709602307.

R. Kurniaty, Ikaningtyas, and P. A. Ruslijanto. Analysis on traditional fishing grounds in indonesia`s natuna waters under international law. IOP Conference Series: Earth and Environmental Science, 137:012039, 2018. doi: 10.1088/1755-1315/137/1/012039.

M-K. Lee, S-H. Moon, Y. Yoon, Y-H. Kim, and B-R. Moon. Detecting anomalies in meteorological data using support vector regression. Advances in Meteorology, 2018:1-14, 06 2018. doi: 10. $1155 / 2018 / 5439256$.

G. Li and J. J. Jung. Dynamic graph embedding for outlier detection on multiple meteorological time series. PLOS ONE, 16(2):1-14, 02 2021. doi: 10.1371/journal.pone.0247119. URL https: //doi.org/10.1371/journal.pone.0247119

F.T. Liu, K. M. Ting, and Z-H. Zhou. Isolation-based anomaly detection. ACM Trans. Knowl. Discov. Data, 6(1), 2012. doi: 10.1145/2133360.2133363.

N. R. Lomb. Least-squares frequency analysis of unequally spaced data. Astrophysics and Space Science, 39:447-462, 1976. doi: https://doi.org/10.1007/BF00648343.

W. McKinney. Data Structures for Statistical Computing in Python. In S. van der Walt and J. Millman, editors, Proceedings of the 9th Python in Science Conference, pages 56 - 61, 2010. doi: 10.25080/Majora-92bf1922-00a.

I. Narulita, F. R. Fajary, M. R. Syahputra, E. Kusratmoko, and M. R. Djuwansah. Spatiotemporal rainfall variability of equatorial small island: case study bintan island, indonesia. Theoretical and Applied Climatology, 144(625-641):625-641, 2021. doi: https://doi.org/10.1007/ s00704-021-03527-x.

W. H. Press, S. A. Teukolsky, W. T. Vetterling, and B. P. Flannery. Numerical Recipes 3rd Edition: The Art of Scientific Computing. Cambridge University Press, USA, 3 edition, 2007. ISBN 0521880688 .

L. Puggini and S. McLoone. An enhanced variable selection and isolation forest based methodology for anomaly detection with oes data. Engineering Applications of Artificial Intelligence, 67:126135, 2018. doi: https://doi.org/10.1016/j.engappai.2017.09.021.

Y. Qin and Y. Lou. Hydrological time series anomaly pattern detection based on isolation forest. In 2019 IEEE 3rd Information Technology, Networking, Electronic and Automation Control Conference (ITNEC), pages 1706-1710, 2019. doi: 10.1109/ITNEC.2019.8729405.

J. Scargle. Studies in astronomical time series analysis. ii - statistical aspects of spectral analysis of unevenly spaced data. The Astrophysical Journal, 263:835 - 853, 1983. doi: 10.1086/160554.

S. Scott and H. Varian. Predicting the present with bayesian structural time series. International Journal of Mathematical Modelling and Numerical Optimisation, 5:4 - 23, 2014. doi: 10.1504/ IJMMNO.2014.059942.

Steven L. Scott. bsts: Bayesian Structural Time Series, 2020. URL https://CRAN.R-project. org/package=bsts. R package version 0.9.5. 
A. Sudirman, J. Mooy, and R. A. Ramadhan M. F. Malufti. Militarising the natuna islands for indonesia's gunboat diplomacy. Central European Journal of International and Security Studies, 13(4):12 - 33, 2013.

M. H. Trauth. Time-Series Analysis, pages 151-213. Springer Berlin Heidelberg, Berlin, Heidelberg, 2015. ISBN 978-3-662-46244-7. doi: 10.1007/978-3-662-46244-7_5.

J. J. Tuite and R. W. Harley. Meteorological and intelligence evidence of long-distance transit of chemical weapons fallout from bombing early in the 1991 persian gulf war. Neuroepidemiology, 40:160 - 177, 2013. doi: 10.1175/1520-0493(1968)096〈0365:ROATMC $\rangle 2.0 . C O ; 2$.

L. Uieda, D. Tian, W. J. Leong, L. Toney, W. Schlitzer, J. Yao, M. Grund, M. Jones, K. Materna, T. Newton, M. Ziebarth, and P. Wessel. PyGMT: A Python interface for the Generic Mapping Tools, March 2021. URL https://doi.org/10.5281/zenodo.4592991.

V.N. Vapnik. An overview of statistical learning theory. IEEE Transactions on Neural Networks, 10 (5):988-999, 1999. doi: 10.1109/72.788640.

G. Wolberg and I. Alfy. Monotonic cubic spline interpolation. pages 188-195, 02 1999. ISBN 0-7695-0185-0. doi: 10.1109/CGI.1999.777953.

P. Xavier, S. Y. Lim, M. F. A. Bin Abdullah, M. Bala, S. N. Chenoli, A. S. Handayani, C. Marzin, D. Permana, F. Tangang, K. D. Williams, and D. J. Yik. Seasonal dependence of cold surges and their interaction with the madden-julian oscillation over southeast asia. Journal of Climate, 33 (6):2467 - 2482, 2020. doi: 10.1175/JCLI-D-19-0048.1.

C. Yao, X. Ma, B. Chen, X. Zhao, and G. Bai. Distribution forest: An anomaly detection method based on isolation forest. In P-C. Yew, P. Stenström, J. Wu, X. Gong, and T. Li, editors, Advanced Parallel Processing Technologies, pages 135-147, Cham, 2019. Springer International Publishing. ISBN 978-3-030-29611-7.

T. Zhang, E. Wang, and D. Zhang. Predicting failures in hard drivers based on isolation forest algorithm using sliding window. Journal of Physics: Conference Series, 1187:042084, 2019. doi: $10.1088 / 1742-6596 / 1187 / 4 / 042084$.

S. Zhong, S. Fu, L. Lin, X. Fu, Z. Cui, and R. Wang. A novel unsupervised anomaly detection for gas turbine using isolation forest. In 2019 IEEE International Conference on Prognostics and Health Management (ICPHM), pages 1-6, 2019. doi: 10.1109/ICPHM.2019.8819409. 